\title{
Intranasal Oxytocin Failed to Affect Chimpanzee (Pan troglodytes) Social Behavior
}

\author{
Darby Proctor ${ }^{1,2, *}$, Sarah E. Calcutt ${ }^{1}$, Kimberly Burke ${ }^{1}$, \& Frans B. M. de Waal ${ }^{1}$ \\ ${ }^{1}$ Living Links, Yerkes National Primate Research Center, Lawrenceville, GA, USA \\ ${ }^{2}$ Department of Psychology, Florida Institute of Technology, Melbourne, FL, USA \\ *Corresponding author (Email: dproctor@fit.edu)
}

Citation - Proctor, D., Calcutt, S. E., Burke, K., \& de Waal, F. B. M. (2016). Intranasal oxytocin failed to affect chimpanzee social behavior. Animal Behavior and Cognition, 3(3), 150-158. doi: 10.12966/abc.04.08.2016

\begin{abstract}
Oxytocin has been suggested as a treatment to promote positive social interactions in people with Autism Spectrum Disorders (ASD). However, it is difficult to test this effect outside of the laboratory in realistic social situations. One way to resolve this issue is to study behavioral changes in closely related species with complex social relationships, such as chimpanzees. Here, we use captive, socially housed chimpanzees to evaluate the effects of oxytocin in a socially complex environment. After administering intranasal oxytocin or a placebo to an individual chimpanzee (total $n=8$ ), she was returned to her social group. An experimenter blind to the condition measured the subject's social behavior. We failed to find a behavioral difference between conditions. As one of the goals for oxytocin administration as a treatment for ASD is increasing prosocial behaviors during 'real world' encounters, it is problematic that we failed to detect behavioral changes in our closest living relatives. However, our null findings may be related to methodological challenges such as determining an effective dose of oxytocin for chimpanzees and how long oxytocin takes to cross the blood-brain barrier. Thus, more research on intranasal oxytocin dosing and uptake are needed to continue exploring whether oxytocin changes social behavior in naturalistic settings and as a treatment for ASD.
\end{abstract}

Keywords - Intranasal oxytocin, Chimpanzee, Social behavior, Autism Spectrum Disorder (ASD)

Since the first study suggested that intranasal oxytocin increases trust in humans (Kosfeld, Heinrichs, Zak, Fischbacher, \& Fehr, 2005), this hormone has captured the attention of both the scientific field and popular press. Naturally occurring oxytocin regulates a variety of behaviors related to sociality in mammals including promoting prosocial behaviors (for a review see: MacDonald \& MacDonald, 2010). Exogenous oxytocin's effect on humans is an important issue in psychology, as intranasal oxytocin administration is one of the few proposed treatments for humans with Autism Spectrum Disorders (ASD; Yatawara, Einfeld, Hickie, Davenport, \& Guastella, 2015). However, as recent reviews have noted, there are problems with the methods and interpretations that intranasal oxytocin promotes positive social interactions (Leng \& Ludwig, 2016; Nave, Camerer, \& McCullough, 2015; Walum, Waldman, \& Young, 2016; Yong, 2015).

One such problem for determining whether oxytocin can be used as a treatment for ASD is that behavioral changes in realistic social contexts are difficult to measure in a lab setting. Instead, some studies rely on caregiver ratings of behavioral changes outside of the lab (Guastella et al., 2015; Yatawara et al., 2015), which may be unreliable. As Guastella et al. (2015) noted, "caregivers who believed their 
child had been assigned the active treatment, regardless of drug assignment, reported greater benefit than those who believed their child received placebo" (p. 449), even though there were no clinically significant results. Thus, studies based on caregiver ratings should be viewed with some skepticism unless the study specifically controls for this bias, as did Guastella and colleagues. Therefore, we need a more rigorous way to test whether oxytocin can affect behavior in "real world" settings.

While there are reports of oxytocin changing animal behavior (see Burkett et al., 2016), these are primarily in laboratory experiments with limited social options. For example, Chang, Barter, Ebitz, Watson, and Platt (2012) found that two hours post intranasal oxytocin administration rhesus macaques (Macaca mulatta) were more likely to provide rewards to their partner in a prosocial task than when administered a placebo. However, results from these types of experiments are not always in the expected direction. In another prosocial task, capuchin monkeys (Cebus apella) decreased the amount of time spent in proximity to their partner and, as a result, shared food less often after being given intranasal oxytocin (Brosnan et al., 2015). Similarly, marmosets (Callithrix penicillata) showed decreased prosocial behavior towards strangers after receiving intranasal oxytocin relative to a placebo (Mustoe, Cavanaugh, Harnisch, Thompson, \& French, 2015). Thus, when social options are restricted to a single partner, we sometimes see an effect of oxytocin on social behavior, although not always in expected ways. While intranasal oxytocin may increase prosocial behaviors in certain situations, we do not yet understand the full scope of how oxytocin affects social behavior.

To our knowledge there have been no observational studies of nonhuman primates given oxytocin and then given free access to their social group. This type of study could help elucidate our understanding of how oxytocin affects social behavior outside of laboratory experiments with limited social options, an important piece of information given the potential use of oxytocin for individuals with ASD. Due both to their close relationship to humans (Steiper \& Young, 2006), as well as their high degree of social complexity (Goodall, 1986), chimpanzees (Pan troglodytes) are an ideal species to test the behavioral effects of oxytocin in a social setting.

Here, we report on a within-subjects study of captive chimpanzees in which one individual was given either intranasal oxytocin or an intranasal placebo (saline). An experimenter blind to the treatment condition then recorded that chimpanzee's social behaviors under the two conditions.

\section{Method}

\section{Subjects}

We tested eight female chimpanzees (age range: $18-35$ years, mean $=25.0$ ) at the Field Station of the Yerkes National Primate Research Center in Lawrenceville, GA, USA. These females were part of a social group of 11 individuals (two males). All females were on birth control (Depo-Provera) that restricted their cycle and none were pregnant or lactating. The subjects were never food or water deprived. They received LabDiet ${ }^{\circledR}$ primate chow, fresh fruits and vegetables multiple times per day and had ad libitum access to water. The chimpanzees were housed in a large outdoor enclosure $\left(711 \mathrm{~m}^{2}\right)$ connected to an indoor building. Oxytocin and saline administration occurred in another building connected to their enclosure used exclusively for research. The Institutional Care and Use Committee of Emory University approved all research (YER-2002759-051217GA).

\section{Procedure}

Chimpanzees were trained to sit and present their nostrils at the mesh of their enclosure. While the chimpanzees presented their nostrils, we used a MABIS NebPak Ultrasonic Nebulizer to administer a $60 \mathrm{IU}$ dose of oxytocin (AgriLabs® $20 \mathrm{IU} / \mathrm{mL}$ ) or an equivalent amount of saline $(3 \mathrm{~mL})$. The intranasal dosage of oxytocin in human studies ranges from 18-60 IU (Heinrichs, Baumgartner, Kirschbaum, \& Ehlert, 2003; MacDonald et al., 2011), with the most common dosage being 20-24 IU (MacDonald et al., 2011), although this is highly variable. We could find no human studies where the dose was adjusted by 
individual weight, so we made no attempt to do this. While chimpanzees weigh, on average, less than an adult human, we expected some oxytocin loss due to the chimpanzees moving their nostrils away from the nebulizer. Thus, we felt the upper limit of the human dosage would be both safe for the chimpanzees and give us the best opportunity for seeing an effect.

Testing was voluntary and at no point were the chimpanzees restrained. The chimpanzees were given diluted juice, a preferred treat, during administration to encourage them to remain stationary. The nebulizer took eight minutes to deliver the full dose. If a chimpanzee moved out of position, the nebulizer was stopped and administration continued when the chimpanzee returned to the correct position. If an individual did not receive the full dose within $15 \mathrm{~min}$, the session was aborted and restarted on another day.

We tested one chimpanzee per day. We counterbalanced the administration order so that half the chimpanzees received oxytocin on their first day of testing and half received nebulized saline. We gave the other compound the following day to control for extraneous variables such as the social environment and weather conditions. Each subject received a total of one oxytocin dose and one saline dose. Following administration, we immediately returned the subject to their social group.

At 30 min post administration, an observer blind to the treatment condition started a 45 -minute focal animal follow that ended 75 min after administration. In human experiments using intranasal oxytocin, $72 \%$ of studies allow between 45 and $60 \mathrm{~min}$ for the oxytocin to uptake (MacDonald et al., 2011). Therefore, by starting at 30 min post administration, we captured the most frequently used windows of oxytocin activation from the human literature.

Table 1

Recorded Behaviors by Sampling Method

\begin{tabular}{ll}
\hline Behavior & Definition \\
\hline $\begin{array}{l}\text { Ad libitum - Frequency } \\
\text { Prosocial }\end{array}$ & Hugs, affiliative touches and kisses \\
Sexual & Sexual inspections, solicitation and mounting behaviors \\
Pant-grunts & Initiating or receiving pant-grunts \\
Agonism & By level of aggression: \\
& $1 . \quad$ Non-contact such as directed display \\
& $3 . \quad$ Contact such as slaps and tugs \\
Movement & Subject moves 6 m or from one indoor run to another \\
Self-grooming & 5 s or more actively grooming self \\
Ad libitum - Duration & \\
Social Proximity & 5 s or more within 2 meters of or in the same indoor run as a group mate \\
Allogrooming & 5 s or more actively grooming or receiving grooming from a group mate \\
Social Play & Any duration of social play behavior \\
Scan - Frequency & Either moving or actively manipulating something with hands or mouth \\
Sleep & Within 2 m of or in the same indoor run as a group mate \\
Active &
\end{tabular}

During observations, we recorded all social interactions measured as either durations or frequencies, including which individual initiated and ended each encounter. The focal animal could simultaneously be involved in multiple social encounters (e.g., being in proximity to one individual while 
grooming another individual). We also recorded self-grooming because injected oxytocin has been reported to increase self-grooming in rats (Ayers, Missig, Schulkin, \& Rosen, 2011; Drago, Pedersen, Caldwell, \& Prange, 1986). Finally, we recorded the activity level of the subject via both frequencies of movement bouts as well as three-minute scan samples of the state of our focal animal (sleeping, active, inactive) and whether they were in proximity to another individual. Table 1 provides a list of the behavioral measures and their definitions.

\section{Results}

All analyses were conducted with SPSS Version 23. When comparing the oxytocin and saline conditions, we failed to find any significant differences between conditions. For example, chimpanzees spent similar amounts of time in proximity to other individuals (Wilcoxon Signed-ranks Test $Z=1.06$, $p=0.16, r=0.27)$, grooming others $(Z=0.11, p=0.92, r=0.03)$, had unchanged numbers of grooming partners $(Z=1.06, p=0.29, r=0.27)$, bouts of self-grooming $(Z=0.95, p=0.34, r=0.24)$ and were active in similar numbers of scan samples $(Z=0.68, p=0.50, r=0.17)$. No behaviors that we measured were significantly different. See Tables $2-4$ for individual data and results of all analyses and Figure 1 for a comparison of grooming behaviors. Therefore, we failed to reject our null hypothesis.

In case we were incorrect about how long the oxytocin took to uptake and based on the recent findings indicating chimpanzees have a slower basal metabolic rate than humans (Pontzer et al., 2016), we then looked only at the final $30 \mathrm{~min}$ of our behavioral observations (beginning 45 minutes postadministration). Again, we failed to find differences between the oxytocin and saline condition. Chimpanzees spent similar amounts of time in proximity to other individuals $(Z=1.12, p=0.26$, $r=0.28)$, grooming others $(Z=0.67, p=0.5, r=0.17)$, had unchanged numbers of grooming partners $(Z$ $=1.52, p=0.13, r=0.38)$, bouts of self-grooming $(\mathrm{Z}=1.16, p=1.16, r=0.29)$ and were active in an exactly equal number of scans $(Z=0, p=1, r=0)$. As with the original analysis, no other behaviors that we measured differed between oxytocin and saline conditions. Thus, in a blind experiment, we failed to observe behavioral differences in chimpanzees that had been given intranasal oxytocin compared to a saline placebo.

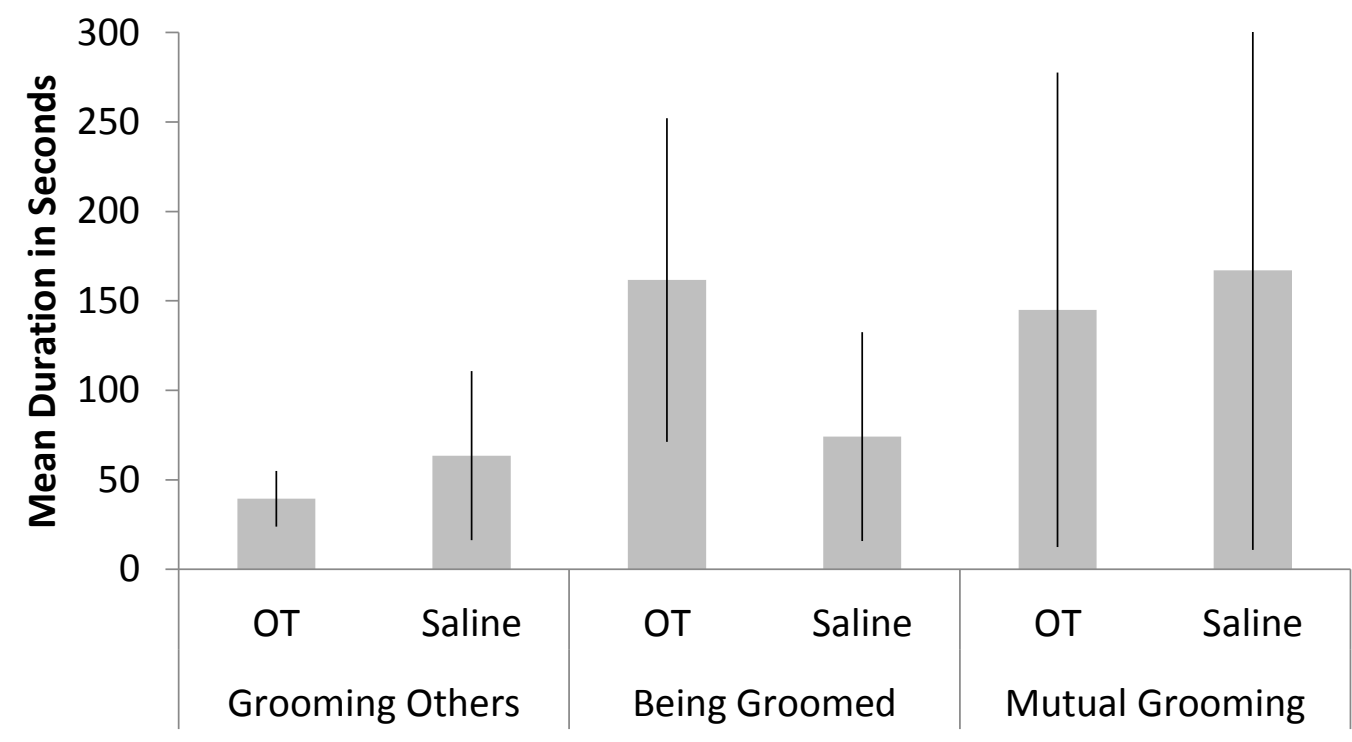

Figure 1. Mean duration of grooming behaviors by condition. We failed to find significant differences between our oxytocin (OT) and saline conditions in regards to any social grooming behaviors. There was a high degree of individual variation, as indicated by the SEM bars. 
Table 2

Frequencies of Behaviors

\begin{tabular}{|c|c|c|c|c|c|c|c|c|c|c|c|c|}
\hline \multirow[b]{2}{*}{ Subject } & \multicolumn{2}{|c|}{ Prosocial Behaviors } & \multicolumn{2}{|c|}{ Self-grooming } & \multicolumn{2}{|c|}{ Sexual Behaviors } & \multicolumn{2}{|c|}{ Movement } & \multicolumn{2}{|c|}{ Grooming Partners } & \multicolumn{2}{|c|}{ Individuals in Proximity } \\
\hline & OT & Saline & OT & Saline & OT & Saline & OT & Saline & OT & Saline & OT & Saline \\
\hline Azalea & 3 & 5 & 6 & 5 & 0 & 0 & 3 & 0 & 3 & 2 & 9 & 5 \\
\hline Barbi & 0 & 2 & 2 & 4 & 0 & 0 & 0 & 2 & 0 & 2 & 5 & 9 \\
\hline Dara & 4 & 0 & 2 & 5 & 0 & 0 & 0 & 2 & 2 & 0 & 6 & 8 \\
\hline Fiona & 0 & 0 & 1 & 8 & 0 & 0 & 2 & 0 & 0 & 1 & 9 & 6 \\
\hline Katie & 0 & 1 & 2 & 2 & 1 & 0 & 1 & 1 & 0 & 0 & 6 & 4 \\
\hline Liza & 1 & 0 & 1 & 1 & 0 & 0 & 2 & 1 & 2 & 0 & 7 & 6 \\
\hline Rita & 1 & 1 & 0 & 2 & 0 & 0 & 1 & 0 & 0 & 0 & 5 & 3 \\
\hline Rowena & 3 & 0 & 9 & 4 & 1 & 1 & 3 & 0 & 3 & 0 & 8 & 5 \\
\hline$M$ & 1.50 & 1.13 & 2.88 & 3.88 & 0.25 & 0.13 & 1.50 & 0.75 & 1.25 & 0.63 & 6.88 & 5.75 \\
\hline$S D$ & 1.5 & 1.62 & 2.85 & 2.09 & 0.43 & 0.33 & 1.12 & 0.83 & 1.30 & 0.86 & 1.54 & 1.85 \\
\hline$Z$ & \multicolumn{2}{|c|}{0.42} & \multicolumn{2}{|c|}{0.95} & \multicolumn{2}{|c|}{1.00} & \multicolumn{2}{|c|}{1.03} & \multicolumn{2}{|c|}{1.06} & \multicolumn{2}{|c|}{1.06} \\
\hline$p$ & \multicolumn{2}{|c|}{0.67} & \multicolumn{2}{|c|}{0.34} & \multicolumn{2}{|c|}{0.32} & \multicolumn{2}{|c|}{0.31} & \multicolumn{2}{|c|}{0.29} & \multicolumn{2}{|c|}{0.29} \\
\hline$r$ & \multicolumn{2}{|c|}{0.11} & \multicolumn{2}{|c|}{0.24} & \multicolumn{2}{|c|}{0.25} & \multicolumn{2}{|c|}{0.26} & \multicolumn{2}{|c|}{0.27} & \multicolumn{2}{|c|}{0.26} \\
\hline
\end{tabular}


Table 3

Duration of Behaviors (in Seconds)

\begin{tabular}{|c|c|c|c|c|c|c|c|c|c|c|}
\hline \multirow[b]{2}{*}{ Subject } & \multicolumn{2}{|c|}{ Proximity } & \multicolumn{2}{|c|}{$\underline{\text { Grooming Others }}$} & \multicolumn{2}{|c|}{ Being Groomed } & \multicolumn{2}{|c|}{$\underline{\text { Mutual Grooming }}$} & \multicolumn{2}{|c|}{ Play } \\
\hline & $\mathrm{OT}$ & Saline & OT & Saline & OT & Saline & OT & Saline & OT & Saline \\
\hline Azalea & 10430 & 9288 & 103 & 410 & 338 & 504 & 1137 & 1337 & 0 & 93 \\
\hline Barbi & 10261 & 7636 & 0 & 82 & 0 & 89 & 0 & 0 & 0 & 0 \\
\hline Dara & 8197 & 10666 & 72 & 0 & 84 & 0 & 0 & 0 & 0 & 0 \\
\hline Fiona & 13078 & 9984 & 0 & 16 & 0 & 0 & 0 & 0 & 0 & 0 \\
\hline Katie & 5709 & 5457 & 0 & 0 & 0 & 0 & 0 & 0 & 16 & 0 \\
\hline Liza & 5497 & 4975 & 104 & 0 & 95 & 0 & 0 & 0 & 0 & 0 \\
\hline Rita & 3762 & 4758 & 0 & 0 & 0 & 0 & 0 & 0 & 0 & 0 \\
\hline$\underline{\text { Rowena }}$ & 15294 & 7757 & 36 & 0 & 776 & 0 & 23 & 0 & 0 & 0 \\
\hline$M$ & 9028.50 & 7565.13 & 39.38 & 63.50 & 161.63 & 74.13 & 145.00 & 167.13 & 2.00 & 11.63 \\
\hline$\underline{S D}$ & 3725.86 & 2165.34 & 44.02 & 133.61 & 255.81 & 165.07 & 375.02 & 442.17 & 5.66 & 30.76 \\
\hline$Z$ & \multicolumn{2}{|c|}{1.06} & \multicolumn{2}{|c|}{0.11} & \multicolumn{2}{|c|}{0.41} & \multicolumn{2}{|c|}{0.45} & \multicolumn{2}{|c|}{0.45} \\
\hline$p$ & \multicolumn{2}{|c|}{0.29} & \multicolumn{2}{|c|}{0.92} & \multicolumn{2}{|c|}{0.69} & \multicolumn{2}{|c|}{0.66} & \multicolumn{2}{|c|}{0.66} \\
\hline$r$ & \multicolumn{2}{|c|}{0.26} & \multicolumn{2}{|c|}{0.03} & \multicolumn{2}{|c|}{0.10} & \multicolumn{2}{|c|}{0.11} & \multicolumn{2}{|c|}{0.11} \\
\hline
\end{tabular}

Table 4

State Behaviors by Percentage of Scans

\begin{tabular}{|c|c|c|c|c|c|c|}
\hline \multirow[b]{2}{*}{$\underline{\text { Subject }}$} & \multicolumn{2}{|c|}{ Proximity } & \multicolumn{2}{|c|}{ Sleep } & \multicolumn{2}{|c|}{ Active } \\
\hline & $\mathrm{OT}$ & Saline & $\mathrm{OT}$ & Saline & $\mathrm{OT}$ & Saline \\
\hline Azalea & $100 \%$ & $100 \%$ & $0 \%$ & $0 \%$ & $93.33 \%$ & $93.33 \%$ \\
\hline Barbi & $100 \%$ & $100 \%$ & $53.33 \%$ & $40 \%$ & $20 \%$ & $20 \%$ \\
\hline Dara & $100 \%$ & $100 \%$ & $0 \%$ & $0 \%$ & $26.67 \%$ & $33.33 \%$ \\
\hline Fiona & $100 \%$ & $100 \%$ & $33.33 \%$ & $20 \%$ & $33.33 \%$ & $26.67 \%$ \\
\hline Katie & $93.33 \%$ & $73.33 \%$ & $0 \%$ & $60 \%$ & $13.33 \%$ & $0 \%$ \\
\hline Liza & $100 \%$ & $93.33 \%$ & $80 \%$ & $66.67 \%$ & $13.33 \%$ & $13.33 \%$ \\
\hline Rita & $86.67 \%$ & $93.33 \%$ & $26.67 \%$ & $40 \%$ & $6.67 \%$ & $20 \%$ \\
\hline$\underline{\text { Rowena }}$ & $100 \%$ & $100 \%$ & $0 \%$ & $6.67 \%$ & $60 \%$ & $33.33 \%$ \\
\hline$M$ & $97.50 \%$ & $95.00 \%$ & $24.17 \%$ & $29.17 \%$ & $33.33 \%$ & $30 \%$ \\
\hline$\underline{S D}$ & $4.64 \%$ & $8.66 \%$ & $28.27 \%$ & $24.71 \%$ & $27.49 \%$ & $26.03 \%$ \\
\hline$Z$ & \multicolumn{2}{|c|}{0.82} & \multicolumn{2}{|c|}{0} & \multicolumn{2}{|c|}{0.68} \\
\hline$p$ & \multicolumn{2}{|c|}{0.41} & \multicolumn{2}{|c|}{1} & \multicolumn{2}{|c|}{0.50} \\
\hline$r$ & \multicolumn{2}{|c|}{0.20} & \multicolumn{2}{|c|}{0} & \multicolumn{2}{|c|}{0.17} \\
\hline
\end{tabular}




\section{Discussion}

Chimpanzees did not show behavioral differences after being dosed with intranasal oxytocin compared to saline when allowed to freely interact with their social group. As one of the goals for oxytocin administration as a treatment for ASD is to increase prosocial behaviors during "real world" encounters, it is problematic that we failed to detect behavioral changes in one of our closest living relatives. However, it is quite possible that our null findings are a result of the methodology used and not because oxytocin has no effect on behavior.

In the human and nonhuman primate literature on intranasal oxytocin administration there are no set standards for appropriate dosing and uptake times. Human doses range from 18-60 IU (Heinrichs et al., 2003; MacDonald et al., 2011) and rhesus macaques, who weigh much less than humans, are dosed with 24 or 48 IU (Chang et al., 2012; Parr, Modi, Siebert, \& Young, 2013). While we used the upper human range of $60 \mathrm{IU}$ for the current study, we have no way of knowing if this was an effective dose for chimpanzees. Thus, standards need to be developed for appropriate doses of intranasal oxytocin for both humans and other primates.

Similarly, it is unclear how long after intranasal administration oxytocin becomes effective. While it is generally thought that measuring oxytocin in cerebral spinal fluid is the most accurate measure, the one study to do so in humans only found a significant increase in oxytocin levels 75 minutes after being dosed with 24 IU (Striepens et al., 2013). This may suggest that we are not waiting long enough to see behavioral changes when testing humans and potentially nonhuman primates, although oxytocin uptake is also mediated by other factors such as stress (Brosnan et al., 2015; Gamer, 2010; Heinrichs, von Dawans, \& Domes, 2009) that may play a role when humans undergo the invasive procedure to collect cerebral spinal fluid. In rhesus macaques, intranasal oxytocin increases in cerebral spinal fluid by 35 minutes post administration (Chang et al., 2012), but oxytocin levels were not tracked over time to determine when they peaked nor how long it took to return to baseline levels. Furthermore, Leng and Ludwig (2016) report that in studies where cerebral spinal fluid was taken, at most $0.005 \%$ of the dose was present (see also Modi, Connor-Stroud, Landgraf, Young, \& Parr, 2014), contributing to the uncertainty of what constitutes an effective dose.

In contrast to data from cerebral spinal fluid, blood plasma levels in humans peak $20-30 \mathrm{~min}$ after dosing (Gossen et al., 2012) and changes can be seen in fMRI scans after 30 min (Scheele et al., 2013). These timelines better match the uptake periods allowed in human and nonhuman primate experiments. However, blood plasma levels may not reflect how much oxytocin has actually reached the brain. Therefore, more research needs to be conducted on appropriate uptake periods before solid conclusions can be drawn from studies such as ours, as we were unable to verify how much oxytocin reached the brains of our subjects.

In addition to questions of dosing and uptake time, our study had other limitations. Due to our small sample size, our study is underpowered, as the available number of chimpanzees limited us. This appears to be an issue in the human literature as well. Walum et al. (2016) conducted a meta-analysis of published intranasal oxytocin studies in humans and found that due to the low power of these studies, the false-positive rate is between $84-88 \%$. Once again, this means that for both human and nonhuman primate studies, more research is needed to verify the findings regarding whether intranasal oxytocin can influence social behavior.

Furthermore, our sample was exclusively female because we were unable to train the two males in the group to use the nebulizer. This makes comparing our results to the human literature challenging, as human studies with intranasal administration tend to be male biased. For example, of the 38 studies reviewed by MacDonald and colleagues (2011) 68\% exclusively tested males. Additionally, there is some indication that intranasal oxytocin affects human males and females differently (Rilling et al., 2014). Therefore, our results should not be generalized to males.

As is often the case in science, our findings have left us with more questions than answers. We need more information about how animals, including humans, process intranasal oxytocin before we can be confident of its behavioral effects, particularly if we want to explore intranasal oxytocin as a treatment 
for people with ASD. Animal models may help us to determine dosing better than human models as more outside factors, like stress and the environment, can be controlled with nonhuman animals. Therefore, it may be useful to replicate this study and conduct other studies using oxytocin with other animal species that can then be monitored during complex social relationships.

\section{Acknowledgements}

This research was supported by the Emory University's FIRST program (NIH/NIGMS IRACDA grant no. K12 GM000680), the base grant to the Yerkes National Primate Research Center by the National Center for Research Resources P51RR165 (currently supported by the Office of Research Infrastructure Programs/OD P51OD11132) and the Living Links Center. The YNPRC is fully accredited by the Association for Assessment and Accreditation of Laboratory Animal Care (AAALAC).

\section{References}

Ayers, L. W., Missig, G., Schulkin, J., \& Rosen, J. B. (2011). Oxytocin reduces background anxiety in a fearpotentiated startle paradigm: Peripheral vs central administration. Neuropsychopharmacology, 36, 24882497. doi: http://www.nature.com/npp/journal/v36/n12/suppinfo/npp2011138s1.html

Brosnan, S. F., Talbot, C. F., Essler, J. L., Leverett, K., Flemming, T., Dougall, P., . . Zak, P. J. (2015). Oxytocin reduces food sharing in capuchin monkeys by modulating social distance. Behaviour, 152, 941-961. doi: http://dx.doi.org/10.1163/1568539X-00003268

Burkett, J. P., Andari, E., Johnson, Z. V., Curry, D. C., de Waal, F. B. M., \& Young, L. J. (2016). Oxytocindependent consolation behavior in rodents. Science, 351, 375-378.

Chang, S. W. C., Barter, J. W., Ebitz, R. B., Watson, K. K., \& Platt, M. L. (2012). Inhaled oxytocin amplifies both vicarious reinforcement and self reinforcement in rhesus macaques (Macaca mulatta). Proceedings of the National Academy of Sciences, 109, 959-964. doi:10.1073/pnas.1114621109

Drago, F., Pedersen, C. A., Caldwell, J. D., \& Prange Jr, A. J. (1986). Oxytocin potently enhances novelty-induced grooming behavior in the rat. Brain Research, 368, 287-295. doi: http://dx.doi.org/10.1016/00068993(86)90573-1

Gamer, M. (2010). Does the amygdala mediate oxytocin effects on socially reinfoced learning? Journal of Neuroscience, 30, 9347-9347. doi: 10.1523/JNEUROSCI.2847-10.2010

Goodall, J. (1986). The chimpanzees of Gombe: Patterns of behavior. Cambridge, MA: Belknap.

Gossen, A., Hahn, A., Westphal, L., Prinz, S., Schultz, R. T., Gründer, G., \& Spreckelmeyer, K. N. (2012). Oxytocin plasma concentrations after single intranasal oxytocin administration - A study in healthy men. Neuropeptides, 46, 211-215. doi: http://dx.doi.org/10.1016/j.npep.2012.07.001

Guastella, A. J., Gray, K. M., Rinehart, N. J., Alvares, G. A., Tonge, B. J., Hickie, I. B., . . Einfeld, S. L. (2015). The effects of a course of intranasal oxytocin on social behaviors in youth diagnosed with autism spectrum disorders: A randomized controlled trial. Journal of Child Psychology and Psychiatry, 56, 444-452. doi: 10.1111/jcpp. 12305

Heinrichs, M., Baumgartner, T., Kirschbaum, C., \& Ehlert, U. (2003). Social support and oxytocin interact to suppress cortisol and subjective responses to psychosocial stress. Biological Psychiatry, 54, 1389-1398.

Heinrichs, M., von Dawans, B., \& Domes, G. (2009). Oxytocin, vasopressin, and human social behavior. Frontiers in Neuroendocrinology, 30, 548-557.

Kosfeld, M., Heinrichs, M., Zak, P. J., Fischbacher, U., \& Fehr, E. (2005). Oxytocin increases trust in humans. Nature, 435, 673-676.

Leng, G., \& Ludwig, M. (2016). Intranasal oxytocin: Myths and delusions. Biological Psychiatry, 79, 243-250. doi: http://dx.doi.org/10.1016/j.biopsych.2015.05.003

MacDonald, E., Dadds, M. R., Brennan, J. L., Williams, K., Levy, F., \& Cauchi, A. J. (2011). A review of safety, side-effects and subjective reactions to intranasal oxytocin in human research. Psychoneuroendocrinology, $36,1114-1126$.

MacDonald, K., \& MacDonald, T. M. (2010). The peptide that binds: A systematic review of oxytocin and its prosocial effects in humans. Harvard Review of Psychiatry, 18, 1-21. doi: 10.3109/10673220903523615 
Modi, M. E., Connor-Stroud, F., Landgraf, R., Young, L. J., \& Parr, L. A. (2014). Aerosolized oxytocin increases cerebrospinal fluid oxytocin in rhesus macaques. Psychoneuroendocrinology, 45, 49-57. doi: 10.1016/j.psyneuen.2014.02.011

Mustoe, A. C., Cavanaugh, J., Harnisch, A. M., Thompson, B. E., \& French, J. A. (2015). Do marmosets care to share? Oxytocin treatment reduces prosocial behavior toward strangers. Hormones and Behavior, 71, 8390. doi: http://dx.doi.org/10.1016/j.yhbeh.2015.04.015

Nave, G., Camerer, C., \& McCullough, M. (2015). Does oxytocin increase trust in humans? Critical review of research. Perspectives on Psychological Science, 10, 772-789.

Parr, L. A., Modi, M., Siebert, E., \& Young, L. J. (2013). Intranasal oxytocin selectively attenuates rhesus monkeys' attention to negative facial expressions. Psychoneuroendocrinology, 38, 1748-1756. doi: http://dx.doi.org/10.1016/j.psyneuen.2013.02.011

Pontzer, H., Brown, M. H., Raichlen, D. A., Dunsworth, H., Hare, B., Walker, K., . . Ross, S. R. (2016). Metabolic acceleration and the evolution of human brain size and life history. Nature, 533, 390-392. doi: 10.1038/nature 17654

Rilling, J. K., DeMarco, A. C., Hackett, P. D., Chen, X., Gautam, P., Stair, S., . . Pagnoni, G. (2014). Sex differences in the neural and behavioral response to intranasal oxytocin and vasopressin during human social interaction. Psychoneuroendocrinology, 39, 237-248. doi: http://dx.doi.org/10.1016/j.psyneuen.2013.09.022

Scheele, D., Wille, A., Kendrick, K. M., Stoffel-Wagner, B., Becker, B., Güntürkün, O., . . Hurlemann, R. (2013). Oxytocin enhances brain reward system responses in men viewing the face of their female partner. Proceedings of the National Academy of Sciences, 110, 20308-20313. doi: 10.1073/pnas.1314190110

Steiper, M. E., \& Young, N. M. (2006). Primate molecular divergence dates. Molecular Phylogenetics and Evolution, 41, 384-394.

Striepens, N., Kendrick, K. M., Hanking, V., Landgraf, R., Wüllner, U., Maier, W., \& Hurlemann, R. (2013). Elevated cerebrospinal fluid and blood concentrations of oxytocin following its intranasal administration in humans. Scientific Reports, 3, 3440. doi: 10.1038/srep03440

Walum, H., Waldman, I. D., \& Young, L. J. (2016). Statistical and methodological considerations for the interpretation of intranasal oxytocin studies. Biological Psychiatry, 79, 251-257. doi: http://dx.doi.org/10.1016/j.biopsych.2015.06.016

Yatawara, C. J., Einfeld, S. L., Hickie, I. B., Davenport, T. A., \& Guastella, A. J. (2015). The effect of oxytocin nasal spray on social interaction deficits observed in young children with autism: A randomized clinical crossover trial. Molecular Psychiatry, 1-7. doi: 10.1038/mp.2015.162

Yong, E. (2015). The weak science behind the wrongly named moral molecule. The Atlantic. Retrieved from http://www.theatlantic.com/science/archive/2015/11/the-weak-science-of-the-wrongly-named-moralmolecule/415581/ 